\title{
Aarnos Estado do Pará, em 2004: uma avaliação do Sistema de Informações sobre Mortalidade
}

\author{
Maternal Mortality in the City of Belém, State of Pará, in 2004: \\ an Evaluation on the Mortality Information System
}

Santana Maria Marinho Mota

Secretaria de Vigilância em Saúde, Ministério da Saúde, Belém-PA, Brasil

Silvana Granado N. da Gama

Departamento de Epidemiologia e Métodos Quantitativos em Saúde, Escola Nacional de Saúde Pública Sérgio Arouca, Fundação Oswaldo Cruz, Ministério da Saúde, Rio de Janeiro-RJ, Brasil

Mariza Miranda Theme Filha

Superintendência de Vigilância em Saúde, Secretaria Municipal de Saúde, Rio de Janeiro-RJ, Brasil

\section{Resumo}

Com o objetivo de conhecer a magnitude da mortalidade materna em Belém, capital do Estado do Pará, no ano de 2004, foram investigados todos os óbitos de mulheres em idade reprodutiva residentes no Município, o que possibilitou uma análise da qualidade do Sistema de Informações sobre Mortalidade (SIM) no tocante ao evento estudado. Os resultados revelaram elevado percentual de óbitos evitáveis (78,6\%), com predominância das causas obstétricas diretas (92,8\%). Encontrou-se $50 \%$ de subenumeração de óbito materno no SIM e uma razão de mortalidade materna de 43,0/100.000 nascidos vivos, a qual foi inferior à razão obtida por meio do SIM (53,2/100.000 nascidos vivos), sendo pontuado um fator de correção de 0,85 . Tal diferença é explicada pelo fato de o SIM ter registrado óbitos maternos não confirmados pelo estudo. Concluiu-se que tão-somente a investigação desses óbitos e o acompanhamento do processo de trabalho do SIM são capazes de qualificar as informações e subsidiar adequadamente a elaboração de políticas de saúde que visem à redução da mortalidade materna.

Palavras-chave: mortalidade materna; coeficiente de mortalidade; causa da morte; indicadores.

\section{Summary}

The main objective of this paper was to know the importance of maternal deaths in 2004 in the city of Belém, capital of the State of Pará. All the cases of deaths involving women living in Belém-PA who were in their reproductive age were investigated and as a result it become possible to analyze the quality of the Mortality Information System (SIM) used in the analysis concerning the events being studied. The outcomes of the analysis revealed a high percentage of avoidable deaths (78.6\%), in which most of the cases were related to direct obstetric causes (92.8\%). The maternal mortality ratio was 45.0/100,000 new born, with a 50\% reduction in the records of maternal death in the SIM, in which occurred a correction factor of 0.85. This difference can be explained due to the fact that SIM had registered non-confirmed maternal deaths in the study. It was possible to conclude that only the investigation of these death cases and the follow up of the SIM's work are able to qualify the information and provide proper basis to create new beath policies aiming at the reduction of maternal death.

Key words: maternal mortality; mortality rate; cause of death; indicators.

Endereço para correspondência:

Núcleo do Ministério da Saúde/Pará, Rua Senador Manoel Barata, 869, Sala 401-403, Centro, Belém-PA, Brasil. CEP:66010-140

E-mail:santana.mota@saude.gov.br 


\section{Introdução}

Os índices de mortalidade materna nos países em desenvolvimento ainda permanecem bastante elevados: a cada ano, mais de 500 mil mulheres morrem em consequiência da gravidez ou do parto, em sua maioria por falta ou inadequação de atendimento. ${ }^{1}$

0 valor máximo aceito pela Organização Mundial da Saúde (OMS) para as mortes maternas é de 20 óbitos para cada 100.000 nascidos vivos (NV). ${ }^{2}$ Países desenvolvidos, como Suécia, Dinamarca, Holanda e Estados Unidos da América (EUA), já apresentavam coeficientes menores que 10/100.000 NV ao final da década de $1970 .{ }^{3}$ Atualmente, na maior parte dos países desenvolvidos, as taxas se situam entre $3 \mathrm{e}$ $12 / 100.000 \mathrm{NV}^{4}$

Segundo os dados do Sistema de Informações sobre Mortalidade (SIM) do Ministério da Saúde, referentes ao ano de 2002, a razão de mortalidade materna (RMM) não corrigida foi de 52,7/100.000 NV, $53,7 / 100.000 \mathrm{NV}$ e $100,0 / 100.000 \mathrm{NV}$ para o Brasil, o Estado do Pará e sua capital, a cidade de Belém, respectivamente. ${ }^{5}$

\section{A falta de qualidade das informações nas diversas Regiões brasileiras fez com que o Ministério da Saúde, em 1990, propusesse a aplicação de diferentes fatores de correção para as razões de mortalidade materna obtidas a partir das estatísticas oficiais.}

Como indicador extremamente sensível das condições de vida da população, a mortalidade materna reflete, principalmente, a desarticulação, desorganização e baixa qualidade da assistência prestada à saúde da mulher durante o ciclo gravídico-puerperal. Uma assistência pronta, oportuna e adequada poderia evitar a maioria dessas mortes. ${ }^{6-8}$

As causas diretamente relacionadas à gravidez, ainda que evitáveis em sua maioria, contribuem com o maior número de mortes maternas e foram responsáveis, em 2002, por $76,7 \%$ desses óbitos no Brasil, 90,4\% no Pará e 100,0\% em Belém-PA. ${ }^{5}$
A RMM é o indicador por excelência na medição da mortalidade materna. A baixa qualidade das informações, entretanto, impede que esse indicador traduza a verdadeira magnitude do problema. Estudos revelam que, mesmo em regiões ou países com boa cobertura de registros ${ }^{9}$ ou que se encontram na condição de desenvolvidos, o número de mortes devido à gravidez e suas complicações ainda é subestimado, embora com menor intensidade que nos países em desenvolvimento. ${ }^{10-12}$

A subinformação, ou seja, o preenchimento incorreto da declaração de óbito (DO) e o sub-registro do evento nas estatísticas oficiais são dois fatores que dificultam o monitoramento do nível da mortalidade materna no Brasil. ${ }^{13} 0$ Estado do Paraná, que dispõe de Comitê de Mortalidade Materna (CMM) e corrige os dados das DO a partir da investigação dos casos, encontrou, para 1999, quase o dobro de óbitos maternos quando comparado com o sistema oficial. ${ }^{14}$

A falta de qualidade das informações nas diversas Regiões brasileiras fez com que o Ministério da Saúde, em 1990, propusesse a aplicação de diferentes fatores de correção para as RMM obtidas a partir das estatísticas oficiais. ${ }^{15}$ Posteriormente, o Ministério promoveu pesquisas com o intuito de se construir fatores de correção específicos para as diferentes Regiões, embora não haja consenso, todavia, sobre quais valores são mais adequados às diversas realidades regionais e estaduais: ${ }^{15}$ Tanaka e Mitsuiki (1999) ${ }^{16}$ encontraram, em estudo realizado no ano de 1997, um fator de ajuste para o Brasil de 2,5; já no estudo realizado por Laurenti e colaboradores, ${ }^{17}$ os valores encontrados foram de 1,4 para o Brasil, 1,08 para a Região Norte, 1,76 para a Nordeste, 1,35 para a Sudeste, 1,83 para a Sul e 1,10 para a Centro-Oeste.

Entre as estratégias governamentais brasileiras para dimensionar o problema e identificar seus determinantes, estão: a Portaria MS n ${ }^{0} 653$, publicada na edição do Diário Oficial da União de 30 de maio de 2003, que obriga, de forma compulsória, as Secretarias de Estado e Municipais a notificarem ao Ministério da Saúde os casos de morte durante e após a gravidez; e a Portaria MS $n^{0} 1.172$, publicada na edição do Diário Oficial da União de 17 de junho de 2004, que estabelece a vigilância epidemiológica da mortalidade infantil e materna como uma das atribuições do Município, cabendo a ele garantir estrutura e equipes compatíveis com o exercício dessas atividades. 
Não obstante a importância do acompanhamento dos óbitos maternos e os dispositivos legais existentes, Belém-PA não possui CMM e sua Secretaria Municipal de Saúde não realiza a investigação dos óbitos de mulheres em idade fértil, tampouco dos óbitos maternos declarados no SIM.

0 presente estudo tem como objetivo estimar a subenumeração dos óbitos maternos no Município de Belém-PA, calcular o fator de ajuste da RMM e comparar os casos registrados no SIM com aqueles identificados pela pesquisa.

\section{Metodologia}

Trata-se de um estudo descritivo de base populacional que investigou os óbitos de mulheres em idade fértil (10 a 49 anos) buscando detectar a presença de gravidez no momento do óbito ou nos 12 meses que 0 antecederam, para, então, identificar e investigar o óbito materno, assim classificado segundo o conceito definido pela OMS.

Foram selecionadas todas as DO correspondentes aos óbitos de mulheres de 10 a 49 anos de idade ocorridos em 2004 e registrados no SIM como sendo de residentes em Belém-PA. Para garantir a qualidade do estudo, também foram investigados os óbitos de residentes em Belém-PA e ocorridos em outros $\mathrm{Mu}$ nicípios do Estado.

Como todos os óbitos da população de estudo foram trabalhados, além dos óbitos maternos declarados, foram investigados aqueles nos quais as causas de morte poderiam ocultar o estado gestacional ('máscaras'); e os ditos óbitos não maternos, ou seja, resultantes de causas incidentais ou acidentais não relacionadas à gravidez e seu manuseio.

Os pesquisadores realizaram a busca ativa de óbitos no SIM, nos hospitais, no Instituto Médico Legal (IML) local, nos cartórios, cemitérios e funerárias e nos relatórios da Secretaria de Estado da Saúde do Pará. Essa metodologia é recomendada pela OMS e pelo Fundo das Nações Unidas para a Infância (UNICEF) como padrão-ouro para estimar a mortalidade materna., ${ }^{9,14}$

A investigação realizou-se em duas etapas. Na primeira, foi usada a Ficha Confidencial de Notificação e Investigação de Óbitos de Mulheres em Idade Fértil, composta por questões retiradas da DO, do Instrumento de Notificação de Óbito de Mulheres em Idade Fértil do Ministério da Saúde e da Ficha Confidencial de Notificação de Óbito de Mulheres em Idade Fértil. Esta última foi validada por Valongueiro e colaboradores $^{18}$ em Camaragibe, Estado de Pernambuco, no ano 2000.

Com base nas informações coletadas, os óbitos foram inicialmente classificados como: 1) óbito ocorrido durante a gravidez, parto e puerpério (GPP) ou até um ano após o término da gestação; 2) óbito não relacionado com o ciclo gravídico-puerperal; e 3) óbito inconclusivo, ou seja, que apesar da busca de informações, não foi possível classificar em um dos dois grupos anteriores.

$\mathrm{Na}$ segunda etapa da busca ativa, os casos classificados como ocorridos durante a GPP ( $1^{\circ}$ grupo) foram então investigados a partir das partes B, C e D do Instrumento de Investigação Confidencial de Óbito Materno, padronizado pelo Ministério da Saúde e adotado pelos Comitês de Morte Materna - CMM. Nessa etapa, analisou-se a história clínica de cada caso e a identificação original da causa básica do óbito, sua codificação e classificação quanto a se tratar ou não de morte materna. Tais procedimentos foram feitos por um obstetra e um epidemiologista do CMM do Município do Rio de Janeiro-RJ.

Para codificação da causa básica do óbito materno, adotou-se o padrão estabelecido no Manual dos CMM, recomendado e publicado pelo Ministério da Saúde,$^{13}$ que segue as definições da Classificação Estatística Internacional de Doenças e Problemas Relacionados à Saúde-Décima Revisão (CID-10) ${ }^{19}$ Consideraram-se as definições de morte materna por causas obstétricas diretas e indiretas, morte materna tardia e morte materna não obstétrica.

Calculou-se a distribuição percentual das causas de morte materna e sua classificação segundo critérios de evitabilidade, a saber: óbitos evitáveis, aqueles que, nas atuais condições de assistência, recursos e técnicas, não deveriam ocorrer; e óbitos inevitáveis, aqueles que ocorreram ainda que todas as ações e procedimentos adotados houvessem sido corretos e oportunos. ${ }^{13} \mathrm{~A}$ análise da evolução clínica e de todos os procedimentos de condução dos casos, para então proceder a classificação definida, foi realizada pelo obstetra e pelo epidemiologista do CMM do Município do Rio de Janeiro-RJ.

Com base nas informações oriundas do estudo, calculou-se a RMM pela razão entre os óbitos maternos por causas diretas e indiretas, ocorridos até 
42 dias após o término da gestação, e o número de nascidos vivos no mesmo local e ano, multiplicado por 100.000 .

A partir da razão entre o número de óbitos maternos de residentes identificados no estudo e os óbitos maternos registrados no SIM, definiu-se o valor do fator de correção ou fator de ajuste dos dados oficiais sobre mortalidade materna do Município de Belém-PA, para 0 ano de 2004.

0 projeto de investigação foi submetido e aprovado pelo Comitê de Ética da Escola Nacional de Saúde Publica Sérgio Arouca, da Fundação Oswaldo Cruz (ENSP/Fiocruz). Preservou-se o anonimato das instituições e dos casos incluídos no estudo.

\section{Resultados}

A Figura 1 sintetiza todas as etapas da investigação dos óbitos de mulheres em idade fértil e os resultados encontrados. Dos 563 óbitos femininos de 10 a 49 anos de idade trabalhados, 56 (10,1\%) foram excluídos da análise por se tratar de mulheres residentes em outros Municípios e que, equivocadamente, haviam sido registradas como residentes em Belém-PA, fato detectado durante o processo de investigação.

Entre os 56 óbitos de residentes em outro Município, seis eram óbitos maternos, dos quais três constavam no SIM como tal e três estavam subenumerados.

Dos 507 óbitos de residentes em Belém-PA, 483 (95,3\%) encontravam-se registrados no SIM e 24 $(4,7 \%)$ foram identificados pela busca ativa. É importante referir que não foram detectados óbitos maternos no grupo de óbitos captados por busca ativa.

Os 507 óbitos de mulheres em idade fértil representaram $15,1 \%$ dos óbitos femininos. Após a investigação, concluiu-se que 27 (5,3\%) ocorreram durante a gravidez, parto e puerpério - GPP - ou até um ano após o término da gestação, 465 (91,7\%) não estavam relacionados com o ciclo gravídico-puerperal e $15(3,0 \%)$ resultaram inconclusivos quanto a essa relação.

Entre os casos inconclusivos, ressalta-se que, para nove deles, os campos 43 e 44 da DO foram assinalados negativamente; nos seis casos restantes, esses campos não foram preenchidos (estavam em branco). A não-localização dos prontuários dessas mulheres ou o fato de se encontrarem mal preenchidos, aliada aos respectivos familiares não terem sido localizados para fornecer maiores esclarecimentos, fez com que estes autores optassem por não excluir a possibilidade de o óbito ter alguma relação com o ciclo gravídico- puerperal.

Entre os 27 óbitos ocorridos durante a GPP ou até um ano após o término da gestação, 14 (51,97\%) foram classificados como maternos; destes, 11 ocorreram até $042^{\circ}$ dia do puerpério e três após esse período.

Na Tabela 1, observa-se que, das 14 mortes relacionadas com GPP, identificadas e codificadas pelo estudo, em cinco, houve concordância com a causa básica registrada no SIM (casos: 1, 4, 7, 8 e 11); em dois casos, a causa básica foi corrigida, com troca de agrupamento dentro do mesmo capítulo da CID-10 (casos: 12 e 13); e em sete casos, promoveu-se correção, com alteração de capítulo (casos: 2, 3, 5, 6, 9, 10 e 14). Ao término da investigação, as principais causas de óbitos foram reagrupadas, verificando-se 0 predomínio das complicações da hipertensão arterial (Tabela 2).

A subenumeração das mortes foi de $50 \%$, haja vista sete casos identificados pelo estudo como óbito materno não haverem sido informados ao SIM como tal. Deles, quatro ocorreram até 42 dias após o término da gestação e três durante o puerpério tardio. É importante ressaltar que, dos casos subenumerados na DO/SIM, em quatro, fazia-se referência à relação com a GPP. Tais informações, contudo, não foram usadas por ocasião da operacionalização do sistema.

A RMM do estudo foi de 45,0 por $100.000 \mathrm{NV}$, levando-se em conta apenas as mortes ocorridas até $042^{\circ}$ dia de puerpério, passando para 57,3 por $100.000 \mathrm{NV}$ quando consideradas as mortes maternas tardias.

0 fator de correção encontrado para os dados oficiais foi de 0,85 , ou seja, menor que 1 , considerando-se, para tanto, a relação entre os 11 óbitos ocorridos até 42 dias após o término da gravidez - identificados por esta investigação - e os 13 casos registrados no SIM.

A Tabela 3 apresenta a classificação dos 14 óbitos relacionados com o ciclo gravídico-puerperal, segundo os critérios de evitabilidade. Ressalta-se que 13 mortes $(92,8 \%)$ foram devidas a causas diretas e $11(78,6 \%)$ consideradas evitáveis, por integrantes de Comitê de Óbito Materno do Município do Rio de Janeiro-RJ. 


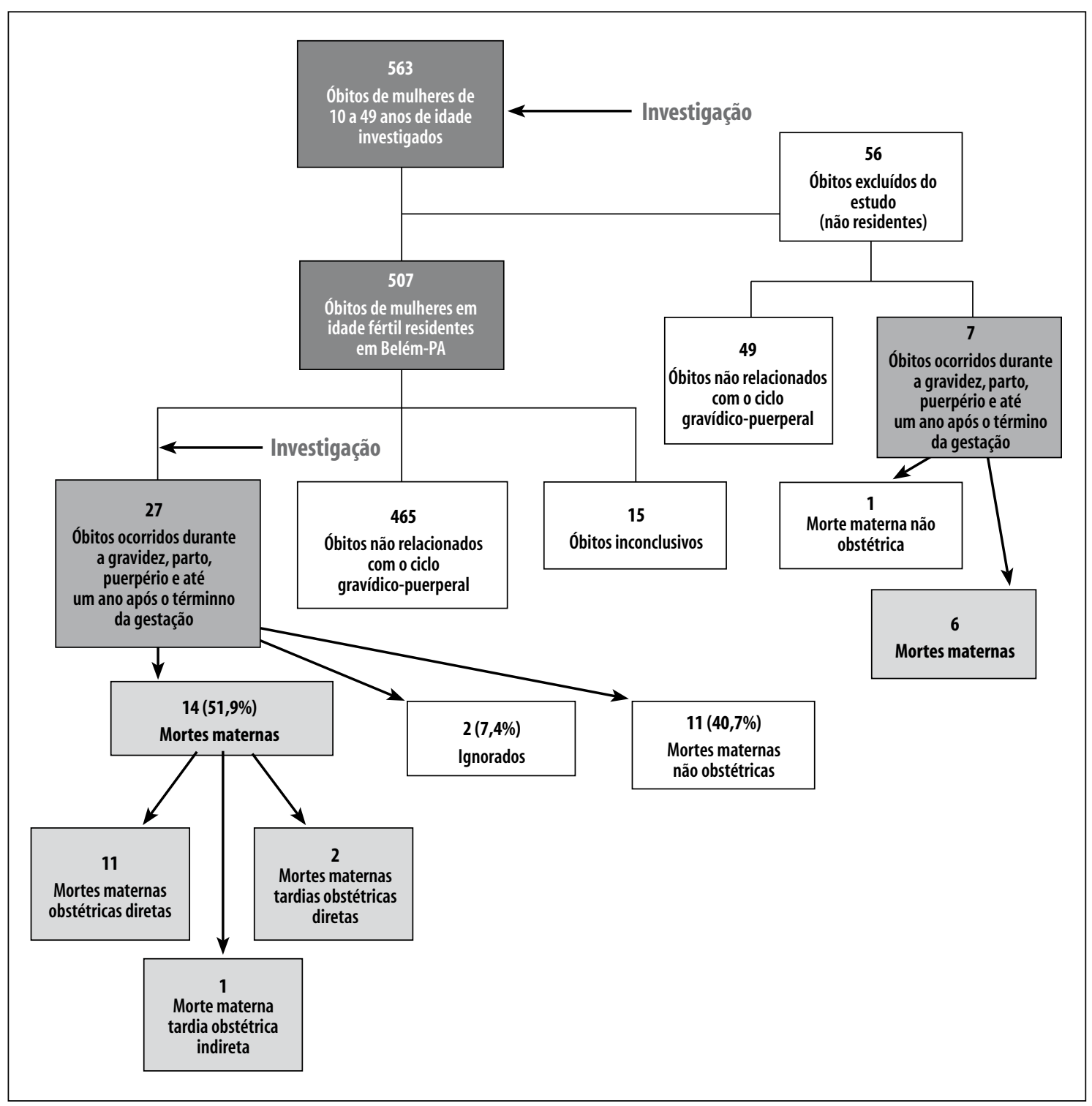

Figura 1 - Fluxograma de óbitos de mulheres em idade fértil investigados em Belém-PA. Brasil, 2004

\section{Discussão}

Tanaka e Mitsuiki ${ }^{16}$ apontam a situação que neste estudo se confirma: a capital do Estado do Pará, pólo de referência para a assistência à saúde regional, provavelmente por essa condição, acaba registrando óbitos de pacientes procedentes de outros Municípios.

As mortes por causas maternas representaram a décima causa mortis entre as mulheres em idade reprodutiva em Belém-PA, valor próximo ao citado por Laurenti ${ }^{20}$ para as capitais brasileiras. 0 achado pode indicar que, apesar de consituir sério problema de Saúde Pública, o óbito materno vem se reduzindo ao longo dos anos. Outra pesquisa realizada no Pará, no ano de 1997, verificou que esses óbitos ocupavam a sexta colocação entre as causas de morte feminina. ${ }^{16}$

Em consonância com outras pesquisas, ${ }^{16,17,21,22}$ ademais, a hipertensão arterial mantém-se como causa principal de morte materna em Belém-PA $(28,6 \%)$, comportamento observado mesmo em países que já alcançaram bons resultados na prevenção do óbito materno, caso do Chile ${ }^{23}$ do Canadáa ${ }^{24}$ dos EUA. ${ }^{25}$ Está claro que a implantação de medidas adequadas para 
Tabela 1 - Situação dos óbitos maternos segundo a causa (CID-10) a identificada na pesquisa e a registrada no Sistema de Informações sobre Mortalidade em Belém-PA. Brasil, 2004

\begin{tabular}{|c|c|c|c|c|}
\hline $\begin{array}{l}\text { Casos } \\
\text { de óbito } \\
\text { materno }\end{array}$ & CID-10 a & Causa básica do estudo & CID-10 ${ }^{a}$ & Causa básica no SIM \\
\hline 1 & 085 & Infecção puerperal & 086.4 & $\begin{array}{l}\text { Febre de origem desconhecida } \\
\text { subsequente ao parto }\end{array}$ \\
\hline 2 & $085-096$ & Infecção puerperal - morte materna tardia. & K 65.8 & Outras peritonites \\
\hline 3 & $085-096$ & Infecção puerperal - morte materna tardia. & N 70.9 & Salpingite e ooforite não especificada \\
\hline 4 & 005.5 & $\begin{array}{l}\text { Outros tipos de aborto, completo ou não } \\
\text { especificado, complicado por infecção do trato } \\
\text { genital ou dos órgãos pélvicos. }\end{array}$ & 002.1 & Aborto retido \\
\hline 5 & 005.5 & $\begin{array}{l}\text { Outros tipos de aborto, completo ou não } \\
\text { especificado, complicado por infecção do trato } \\
\text { genital ou dos órgãos pélvicos. }\end{array}$ & A 41.9 & Septicemia não especificada \\
\hline 6 & 005.5 & $\begin{array}{l}\text { Outros tipos de aborto, completo ou não } \\
\text { especificado, complicado por infecção do trato } \\
\text { genital ou dos órgãos pélvicos. }\end{array}$ & A 41.9 & Septicemia não especificada \\
\hline 7 & 014.1 & Pré-eclâmpsia grave & 011 & $\begin{array}{l}\text { Distúrbio hipertensivo pré-existente } \\
\text { com proteinúria superposta }\end{array}$ \\
\hline 8 & 014.1 & Pré-eclâmpsia grave & 014.0 & Pré-eclâmpsia moderada \\
\hline 9 & 015.0 & Eclâmpsia na gravidez & Ј 18.9 & Pneumonia não especificada \\
\hline 10 & 011 & $\begin{array}{l}\text { Distúrbio hipertensivo pré-existente com } \\
\text { proteinúria superposta }\end{array}$ & D 65 & Coagulação intravascular disseminada \\
\hline 11 & 000.1 & Gravidez tubária & 000.1 & Gravidez tubária \\
\hline 12 & 062.2 & Outras formas de inércia uterina & 047.9 & Falso trabalho de parto não especificado \\
\hline 13 & 075.4 & $\begin{array}{l}\text { Outras complicações de procedimentos ou de } \\
\text { cirurgia obstétrica }\end{array}$ & 014.9 & Pré-eclâmpsia não especificada \\
\hline 14 & $099.8-096$ & $\begin{array}{l}\text { Outras doenças e afecções especificadas } \\
\text { complicando a gravidez, o parto e o puerpério } \\
\text { - morte materna tardia. }\end{array}$ & A 16.2 & $\begin{array}{l}\text { Tuberculose pulmonar, sem menção } \\
\text { de confirmação bacteriológica ou } \\
\text { histológica. }\end{array}$ \\
\hline TOTAL & & 14 & & 7 \\
\hline
\end{tabular}

Fonte:Sistema de Informações sobre Mortalidade (SIM)/Secretaria Municipal de Saúde de Belém-PA; e dados do estudo. a) CID-10: Classificação Estatística Internacional de Doenças e Problemas Relacionados à Saúde - Décima Revisão 
Tabela 2 - Distribuição dos óbitos maternos identificados na pesquisa, segundo causa (CID-10), em Belém-PA. Brasil, 2004

\begin{tabular}{lc}
\hline Causas & No \\
\hline Hipertensão arterial $(0$ 14.1; $15.0 ; 011)$ & 4 \\
Aborto $(0$ 05.5) & 3 \\
Infecção puerperal $(0$ 85; 085 - 0 96) & 3 \\
Gravidez tubária (0 00.1) & 1 \\
Outras formas de inércia uterina (0 62.2) & 1 \\
Outras complicações de procedimentos ou de cirurgia obstétrica (0 75.4) & 1 \\
Outras doenças e afeçõoes especificadas complicando a gravidez, o parto e o puerpério (0 99.8 - 096$)$ & 1 \\
\hline TOTAL & 14 \\
\hline
\end{tabular}

a) CID-10:Classificação Estatística Internacional de Doenças e Problemas Relacionados à Saúde - Décima Revisão

Tabela 3 - Distribuição dos óbitos maternos de residentes em Belém-PA ocorridos até um ano após o término da gestação, segundo tipo e evitabilidade. Brasil, 2004

\begin{tabular}{lcccc}
\hline & Evitável & Provavelmente evitável & Inevitável & Total \\
\cline { 2 - 5 } Tipo & $\mathrm{N}^{0}$ & $\mathrm{~N}^{\circ}$ & $\mathrm{N}^{\circ}$ & $\mathbf{N}^{\circ}$ \\
\hline Óbito materno obstétrico direto & 9 & 1 & 1 & 11 \\
Óbito materno tardio obstétrico direto & 2 & 0 & 0 & 2 \\
Óbito materno tardio obstétrico indireto & 0 & 0 & 1 & 1 \\
\hline TOTAL & $11(78,6 \%)$ & $\mathbf{1 ( 7 , 1 \% )}$ & $\mathbf{2 ( 1 4 , 3 \% )}$ & $\mathbf{1 4}(100,0 \%)$ \\
\hline
\end{tabular}

reduzir a mortalidade materna deve considerar que, quase sempre, essa redução é gradual. ${ }^{20}$

Complicações do aborto e infecções puerperais também foram importantes causas de óbitos maternos em 2004. Essas questões evidenciam que a morte materna é um relevante problema de saúde, não só pela magnitude como pela evitabilidade que envolve suas causas, traduzindo-se no acesso ao Sistema Único de Saúde e na pouca eficácia de seus serviços, na frequiência às consultas do pré-natal e na assistência ao parto e puerpério.

A predominância de causas diretas e evitáveis mediante ações direcionadas ao planejamento familiar, gravidez, parto e puerpério mostra ainda quão frágil é, todavia, a atenção dispensada a esse segmento da população, quando se considera a disponibilidade e qualidade dos recursos de saúde existentes. ${ }^{17,21,26}$

0 sub-registro em cartório, fato constatado para algumas cidades brasileiras, ${ }^{16}$ não foi detectado no presente estudo. Já a existência de óbitos que, apesar de registrados em cartório, não se encontravam notificados no SIM, denota falha no fluxo das DO, possivelmente agravada pela interposição das funerárias na rotina funcional dos óbitos domiciliares, devendo-se proceder a averiguação dessa possibilidade, para que sejam efetuados os ajustes necessários. 
A diferença entre os dados obtidos após investigação e os disponíveis no SIM revelou a elevada subenumeração dos óbitos ocorridos em alguns momentos do ciclo gravídico-puerperal, evidência também identificada por outros estudos. . $11,12,17,27,28^{2}$

À semelhança do encontrado por Parpinelli e colaboradores ${ }^{27}$ para a cidade de Campinas, Estado de São Paulo, a maior subenumeração observou-se no grupo relacionado às complicações do aborto e do puerpério. Para o restante dos casos, principalmente por se tratar de óbitos maternos tardios, a subenumeração no SIM foi consequiência do incorreto preenchimento da DO. Isso acontece quando o médico, ao preencher o documento, registra apenas o diagnóstico ou complicação da patologia associada à gravidez e omite o passado gestacional - talvez, por desconhecer sua influência sobre 0 agravamento da doença existente ou não lhe dar a devida importância.

0 atual estudo evidencia 0 quanto é necessário, além do correto preenchimento da DO, o investimento na supervisão do processo de trabalho relacionado à codificação, digitação e elaboração de relatórios emitidos pelo SIM.

É importante ressaltar que as falhas detectadas em Belém-PA refletem-se, efetivamente, na qualidade das informações do Estado do Pará. Segundo dados da Secretaria de Estado da Saúde, em 2004, o SIM registrou 79 óbitos maternos - que passariam a ser 89, caso os dez óbitos subenumerados - sete de Belém e três de outros Municípios - fossem corretamente computados.

Comparativamente às RMM de outras cidades brasileiras, entre 50 e $80 / 100.000 \mathrm{NV},{ }^{17,21,26,28}$ a mortalidade materna encontrada em Belém-PA foi inferior - 45/100.000 NV -; e praticamente igual à referida por Parpinelli e colaboradore ${ }^{27}$ para a cidade de Campinas-SP (42,2/100.000 NV).

Diferentemente do esperado diante da elevada subenumeração detectada, a RMM de Belém-PA $(45,0 / 100.000 \mathrm{NV})$ foi inferior à calculada com base tão-somente nos dados do SIM $(53,2 / 100.000 \mathrm{NV})$. 0 fator de correção ou de ajuste foi negativo $(0,85)$, bem menor que o observado em pesquisas realizadas sobre outros Municípios brasileiros. ${ }^{16,17,27,28}$

Considerando o resultado contraditório referido e o fator de correção pontuado resultante da relação entre $o$ dado da pesquisa e o originalmente existente no SIM, estes pesquisadores optaram por aprofundar o estudo e analisar cada um dos óbitos registrados no sistema como maternos. Verificaram que a situação descrita é fruto da invasão de óbitos maternos no SIM/Belém-PA: alguns, erroneamente codificados e registrados originalmente como sendo maternos; outros, ainda que maternos, na verdade procedentes de outros Municípios. Tais óbitos compensaram, numericamente, a subenumeração dos sete óbitos maternos de residentes em Belém-PA e contribuíram para que o valor da RMM do SIM fosse superior ao registrado pelo estudo.

Este achado pode caracterizar um resultado pouco comum, ${ }^{29}$ merecedor de outras avaliações. 0 fato é que, se numericamente, o fator de ajuste apresenta potencial para corrigir os dados oficiais, não acompanha as mudanças na qualidade das informações decorrentes do nível de organização que o sistema pode alcançar. Assim, as inconsistências internas não são resolvidas. 0 fator de correção, portanto, não é suficiente para detectar os verdadeiros casos de forma a qualificar as informações sobre os óbitos maternos e apontar os determinantes do processo desse evento. Esse propósito somente será alcançado com a implantação da investigação rotineira dos óbitos de mulheres em idade reprodutiva.

Torna-se evidente que as informações sobre óbito materno no SIM são inconsistentes. Estudos focados na morte materna devem ser estimulados, portanto, e o conhecimento advindo sobre suas causas e verdadeira magnitude definirão o ponto de partida para 0 desenvolvimento de uma política adequada de combate à mortalidade materna. ${ }^{30}$

Nesse sentido, dada a importância do problema, um encaminhamento mais resolutivo - do que a realização de estudos com objetivo de calcular fatores de ajuste - será a convocação dos Municípios para o desenho e implementação de sistemas de vigilância ativos, de contínua e sistemática coleta de dados, capazes de melhor retratar a mortalidade materna. ${ }^{24,31}$

Como parte integrante da estrutura do sistema de vigilância, a ação do Comitê de Mortalidade Materna - CMM - constitui uma das estratégias existentes para a superação da precariedade de informações disponíveis e das dificuldades na obtenção de dados consistentes sobre a morte materna em nosso país.

Como instrumento político e de controle social, $o$ CMM assume tal dimensão que lhes confere status, mais além de uma estratégia de vigilância epidemiológica, de instância de participação da sociedade na 
proposição das medidas para a prevenção e redução da morte materna. ${ }^{32}$

É pouco provável que medidas técnicas, exclusivamente, levem à redução da mortalidade materna. Faz-se necessário o comprometimento das autoridades políticas $^{12}$ com a questão, colocando-a à altura das prioridades da Saúde e encarregando às Secretarias de Estado e Municipais a responsabilidade pelo estímulo à criação, promoção e garantia de condições para que os Comitês de Mortalidade Materna sejam atuantes, capacitados mediante treinamentos periódicos, organizados de forma descentralizada e coordenados por um CMM no nível central.

É mister que Belém-PA invista na implantação de seu Comitê de Mortalidade Materna. Ele deve se constituir em um sistema de vigilância da morte materna, visando

\section{Referências bibliográficas}

1. Sen G. Meio milhão de grávidas morre sem atendimento. Radis - Comunicação em Saúde 2004; 24:5.

2. Theme-Filha MM, Silva RI, Noronha CP. Mortalidade materna no Município do Rio de Janeiro, 1993-1996. Cadernos de Saúde Pública 1999;15(2):397-403.

3. Laurenti R, Mello-Jorge MHP, Lebrão ML, Gotlieb SLD. Estatísticas de saúde. $2^{\mathrm{a}}$ ed. rev. e atual. São Paulo-SP: Editora Pedagógica e Universitária; 1987.

4. Laurenti R. Perfil da mortalidade materna. In: Minayo MCS, organizador. Os Muitos brasis - saúde e população na década de 80. São Paulo-SP: Hucitec; 1995; p. 304-319.

5. Ministério da Saúde. Informações de saúde [dados na Internet]. Brasília: Ministério da Saúde. [acessado 2005 jun. 7]. Disponível em: http://www.saude.gov. $\mathrm{br} /$ datasus

6. Tanaka AC, organizador. Saúde da mulher e direitos reprodutivos: dossiê mortalidade materna. São PauloSP: Rede Nacional Feminista de Saúde e Direitos Reprodutivos - Rede Saúde; 2001.

7. Silva KS, D’orsi E, Lowndes CM, Reis ACCV. A mortalidade materna no Brasil no período 19801993. In: Giffin K, Costa SH, organizadores. Questões da saúde reprodutiva. Rio de Janeiro-RJ: Fiocruz; 1999. cap. 12. p. 205-225.

8. AbouZahr C, Wardlaw T, Staton C, Hill K. Maternal mortality. World Health Statistic Quartely 1996;49(2):77-87. não apenas à melhoria da certificação do óbito como também ao aprimoramento do processo de trabalho previsto pelo SIM, para aprimorar o conhecimento sobre o problema e, dessa forma, permitir a proposição de atividades que tenham por meta 0 êxito da prevenção da mortalidade associada à gravidez.

A limitação do estudo consistiu na existência de dois óbitos ocorridos durante a GPP, classificados como ignorados quanto ao fato de serem ou não óbitos maternos. Suas informações disponíveiss nas fontes consultadas não foram suficientes para assegurar causas relacionadas com ou agravadas pela gravidez, ou ainda, por procedimentos de atenção a ela. A limitação inicial diante dos 15 óbitos inconclusivos foi atenuada pelo fato de os campos 43 e 44 das D0 não estarem assinalados positivamente.

9. Laurenti R, Mello-Jorge MHP, Gotlieb SLD. Reflexões sobre a mensuração da mortalidade materna. Cadernos de Saúde Pública 2000;16(1):23-30.

10. Koonin LM, MacKay AP, Berg CJ, Atrash HK, Smith JC. Pregnancy-related mortality surveillance-United States, 1987-1990. MMWR CDC Surveillance Summaries 1997 Aug;46(4):17-36.

11. Atrash HK, Alexander S, Berg CJ. Maternal mortality in developed countries: not just a concern of the past. Obstetrics and Gynecology 1995 0ct;86(4 Pt 2): 700-705.

12. Bouvier-Colle MH, Varnoux N, Costes P, Hatton F. Reasons for the underreporting of maternal mortality in France, as indicated by a survey of all deaths of women of childbearing age. International Journal of Epidemiology 1991;20:717-721.

13. Ministério da Saúde. Manual dos comitês de mortalidade materna. $2^{\mathrm{a}}$ ed. Brasília: MS; 2002.

14. Laurenti R. Medindo a mortalidade materna. São Paulo-SP: Centro Brasileiro de Classificação de Doenças/Universidade de São Paulo; 2001.

15. Secretaria de Vigilância em Saúde. Saúde Brasil 2004: uma análise da situação de saúde. Evolução da mortalidade materna no Brasil. Brasília-DF: Ministério da Saúde; 2004. p. 85-133.

16. Tanaka AC, Mitsuiki L. Estudo da magnitude da mortalidade materna em 15 cidades brasileiras: 
relatório de pesquisa. São Paulo-SP: Universidade de São Paulo; 1999.

17. Laurenti R, Mello-Jorge MHPM, Gotlieb SLD. A mortalidade materna nas capitais brasileiras: algumas características e estimativas de um fator de ajuste. Revista Brasileira de Epidemiologia 2004;7(4): 449-460.

18. Valongueiro S, Ludermir AB, Gominho LAF. Avaliação de procedimentos para identificar mortes maternas. Cadernos de Saúde Pública 2003;19supl:2.

19. Organização Mundial da Saúde. Classificação estatística internacional de doenças e problemas relacionados à saúde. $10^{a}$ revisão. $9^{a}$ ed. rev. São Paulo-SP: Editora da Universidade de São Paulo; 2003. V 1.

20. Laurenti R. Mortalidade materna: desafios para sua redução e a questão da mensuração e coleta de dados. Apresentação feita na Eurolac Conference; 2004 abr.16; Recife-PE, Brasil.

21. Costa AAR, Ribas MSSS, Amorim MMR, Santos LC. Mortalidade materna na cidade do Recife. Revista Brasileira de Ginecologia e Obstetrícia 2002;24(7):455-462.

22. Cecatti JG, Albuquerque RM, Hardy E, Faúndes A. Mortalidade materna em Recife: causas de óbitos maternos. Revista Brasileira de Ginecologia e Obstetrícia 1998;20(1):7-11.

23. Donoso E. Plan de accion regional para la reduccion de la mortalidad materna en las americas: resultados de Chile. Revista Chilena de Obstetricia y Ginecología 2003;68(1):13-15.

24. Health Canada. Special report on maternal mortality and severe morbidity in Canada - enhanced surveillance: the path to prevention. Ottawa: Minister of Public Works and Government Services Canada; 2004.
25. Donoso ES, Oyarzún EE. Análisis comparativo de la mortalidad materna en Chile, Cuba y Estados Unidos de Norteamérica. Revista Chilena de Obstetricia y Ginecología 2004;69(1):14-18.

26. Marcus PAF, Veja CEP, Boyaciyan K, Barbosa SA. Estudo da mortalidade materna no Município de São Paulo durante o ano de 1995. Revista Brasileira de Ginecologia e Obstetrícia 1996;18(9):731-736.

27. Parpinelli MA, Faúndes A, Cecatti JG, Surita FGC, Pereira BG, Júnior RP, Amaram E. Subnotificação da mortalidade materna em Campinas: 1992-1994. Revista Brasileira de Ginecologia e Obstetrícia 2000;22(1):27-32.

28. Albuquerque RM, Cecatti JG, Hardy E, Faúndes A. Mortalidade materna em Recife. Avaliação da subenumeração de estatísticas oficiais. Cadernos de Saúde Pública 1997;13:59-65.

29. Ministério da Saúde. Estudo da mortalidade de mulheres de 10 a 49 anos, com ênfase na mortalidade materna: relatório final. Brasília-DF: MS; 2006.

30. Mishima SM. Constituição do gerenciamento local na rede básica de saúde em Ribeirão Preto-SP [Tese de Doutorado]. Ribeirão Preto-SP: Escola de Enfermagem de Ribeirão Preto; 1995.

31. Kestler E, Ramírez L. Pregnancy-related mortality in Guatemala, 1993-1996. Revista Panamericana de Salud Pública 2000 Jan;7(1):414-416.

32. Rodrigues AV, Siqueira AAF. Uma análise da implementação dos comitês de estudos de morte materna no Brasil: um estudo de caso do Comitê do Estado de São Paulo. Cadernos de Saúde Pública 2003;19(1):183-189.

\section{Recebido em 27/09/2006}

Aprovado em 09/10/2007 